\title{
Logic and AI in China: An Introduction
}

\author{
Fenrong Liu $\cdot$ Kaile Su
}

Published online: 20 February 2013

(C) Springer Science+Business Media Dordrecht 2013

The year 2012 has witnessed worldwide celebrations of Alan Turing's 100th birthday. A great number of conferences and workshops were organized by logicians, computer scientists and researchers in AI, showing the continued flourishing of computer science, and the fruitful interfaces between logic and computer science. Logic is no longer just the concept that Frege had about one hundred years ago, let alone that of Aristotle twenty centuries before. One of the prominent features of contemporary logic is its interdisciplinary character, connecting across mathematics, philosophy, modern computer science, and even the cognitive and social sciences.

China is no exception. This special issue explores interfaces of logic with AI and other disciplines, and it attempts to give a bird's eye view of the developing landscape of logic and AI in China. Our contributors come from computer science, AI, philosophy and linguistics, united by their interest in using formal logical tools and notions to deal with substantial problems in their fields. We are aware that AI is a vast area, and that the limitations of space in a volume like this may provide a somewhat incomplete or biased view. Still we hope that the six papers selected here illustrate the concerns and curiosity driving Chinese researchers, on topics shared with our colleagues around the world.

One of the active research areas in AI is planning under uncertainty, with incomplete knowledge, where a well-established logical framework is the action language of Son and Baral (Son and Baral 2001). This language can express the notions of sensing actions and making conditional plans. However, its current semantics is not yet satisfactory, since according to the semantics an agent would

\section{F. Liu (ه)}

Department of Philosophy, Tsinghua University, Beijing, China 100084

e-mail: fenrong@tsinghua.edu.cn

K. Su

Institute for Integrated and Intelligent Systems Griffith University, Bribane 4111, QLD, Australia

e-mail: k.su@griffith.edu.au 
not have knowledge of what happens after a plan is executed, which can be a serious problem in a safety-critical environment. In their paper Proof Systems for Planning under Cautious Semantics (Shen and Zhao 2013), Yuping Shen and Xishun Zhao address this problem and propose a cautious and weakly cautious semantics for the action language. This new semantics enables the agent to know exactly what happens after executing an action. Hoare style proof systems are then proposed and proved to be sound and complete. What is more, the proposed new system reduces the reasoning complexity of the action language.

In Preferential Semantics for Plausible Subsumption in Possibility Theory (Qi and Zhang 2013), Guilin Qi and Zhizheng Zhang investigate another important issue in AI: how to handle exceptions in a knowledge-based system. This has become a hot topic in recent years, especially in ontological design, where description logics are widely used. The question then arises whether we can get a nonmonotonic extension of description logic. To answer this, the authors first propose three preferential semantics for plausible subsumption in the framework of possibility theory (Dubois and Prade 1985) and study their properties and their relationships. Then they introduce preferential subsumptions to a description logic-based knowledge system, and define an entailment relation of plausible subsumption. Moreover, they study reductions of the new semantics to standard semantics for an expressive description logic.

Counterfactual reasoning has been explored extensively by philosophers, cf. Lewis (1973), Stalnaker (1968). But in the last two decades, causal models have been proposed for modeling counterfactual reasoning by researchers in AI. In $A$ Lewisian Logic of Causal Counterfactuals (Zhang 2013), Jiji Zhang studies classes of causal models and provides formal characterizations. In particular, a new logic system is proposed and proved complete for the class of 'Lewisian models' satisfying all the principles of David Lewis's logic of counterfactuals. Moreover, Zhang compares his new framework with those of Lewis and Stalnaker, and also establishes formal connections with more recent approaches by Galles and Pearl, and by Halpern.

Consistency is a fundamental notion in logic, but it also plays a key role in many techniques for solving constraint satisfaction problems (Mackworth 1977). In Variable-Centered Consistency in Model RB (Li et al. 2013), Liang Li, Tian Liu and $\mathrm{Ke} \mathrm{Xu}$ start with a discussion of $i$-consistency in 'Model RB' and 'Model RD', two models of random constraint satisfaction problems with growing domain size, then compare it with the notion of t-consistency and strong t-consistency in well-known earlier work by Freuder. After that, the authors define a new kind of variablecentered consistency, and show an upper bound on a suitably chosen parameter in Model RB and Model RD, such that up to this bound, all typical instances of Model $\mathrm{RB}$ are variable-centered consistent.

Language understanding is a cognitive process that human beings carry out every day. Metaphor is a pervasive phenomenon in natural languages, and its cognitive role calls for interpretation. There is a growing body of literature on cognitive mechanisms underlying metaphor. The paper An Ontology-Based Approach to Metaphor Cognitive Computation (Huang et al. 2013) by Xiaoxi Huang, Huaxin Huang, Beishui Liao, and Cihua Xu proposes a theoretical framework for metaphor 
understanding based on recent results from cognitive science (Shutova 2010). They then introduce ontology as a knowledge representation method, and present a new ontological model to formalize metaphor-based knowledge. An algorithmic description is provided using a quantitative measure of integrated degree, and its effectiveness is demonstrated.

Puzzles and their solution mechanisms are a recurrent theme in many fields. Dynamic epistemic logic (van Ditmarsch et al. 2007); (van Benthem 2011) is a recent framework that can handle information dynamics in knowledge puzzles. In Reasoning About Agent Types and the Hardest Logic Puzzle Ever (Liu and Wang 2013), Fenrong Liu and Yanjing Wang propose a new dynamic-epistemic logic that can specify different types of agents and analyse agents' knowledge, communication and reasoning. To demonstrate its power, Smullyan's well-known Knights and Knaves puzzles are formalized, as well as the Hardest Logic Puzzle Ever (HLPE) of Boolos (Boolos 1996). Moreover, a spectrum of new puzzles is found involving knowledge-based agent types, and their solution complexity is shown to be harder than that for existing logic puzzles. It is also shown that a version of HLPE in which the agents do not know the others' types does not have a solution at all.

Finally, we would like to thank the editor of Minds and Machines, Gregory Wheeler, for his efficient assistance and kind support. We also thank Ties Nijssen at Springer for his encouragement to start this project at the first place. And of course, our appreciation goes to our authors, as well as all the colleagues who have helped us review the papers in this collection.

\section{References}

Boolos, G. (1996). The hardest logic puzzle ever. The Harvard Review of Philosophy, 6, 62-65.

Dubois, D., \& Prade, H. (1985). Possibility theory: An approach to computerized processing of uncertainty. New York, London: Plenum Press.

Huang, X., Huang, H., Liao, B., \& Xu, C. (2013). An ontology-based approach to metaphor cognitive computation. Minds and Michines, 23(1). doi:10.1007/s11023-012-9269-z.

Lewis, D. (1973). Counterfactuals. Oxford: Blackwell.

Li, L., Liu, T., \& Xu, K. (2013). Variable-centered consistency in model RB. Minds and Michines, 23(1). doi:10.1007/s11023-012-9270-6.

Liu, F., \& Wang, Y. (2013). Reasoning about agent types and the hardest logic puzzle ever. Minds and Michines, 23(1). doi:10.1007/s11023-012-9287-x.

Mackworth, A. K. (1977). Consistency in networks of relations. Artificial Intelligence, 8, 99-118.

Qi, G., \& Zhang, Z. (2013). Preferential semantics for plausible subsumption in possibility theory. Minds and Michines, 23(1). doi:10.1007/s11023-012-9300-4.

Shen, Y., \& Zhao, X. (2013). Proof systems for planning under cautious semantics. Minds and Michines, 23(1). doi:10.1007/s11023-012-9288-9.

Shutova, E. (2010). Models of metaphor. In Proceedings of the 48th annual meeting of the association for computational linguistics (pp. 688-697). Uppsala, Sweden.

Son, T.C., \& Baral, C. (2001). Formalizing sensing actions: A transition function based approach. Artificial Intelligence, 125(1-2), 19.

Stalnaker, R. (1968). Studies in logical theory, chapter A theory of conditionals (pp. 98-112). Oxford: Blackwell.

van Benthem, J. (2011). Logical dynamics of information and interaction. Cambridge University Press, Cambridge.

van Ditmarsch, H., van der Hoek, W., \& Kooi, B. (2007). Dynamic epistemic logic. Berlin: Springer. 
Zhang, J. (2013). A lewisian logic of causal counterfactuals. Minds and Michines, 23(1). doi: 10.1007/s11023-011-9261-z. 